\title{
Optimization of Fullerene-based Negative tone Chemically Amplified Fullerene Resist for Extreme Ultraviolet Lithography
}

\author{
A. Frommhold ${ }^{\mathrm{a}}$, D.X. Yang ${ }^{\mathrm{b}}$, A. McClelland ${ }^{\mathrm{c}}$, X. Xue ${ }^{\mathrm{d}}$, Y. Ekinci ${ }^{\mathrm{e}}$, R.E. Palmer ${ }^{\mathrm{b}}$, A.P.G. Robinson ${ }^{* a}$

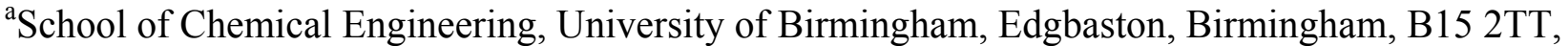 \\ UK \\ ${ }^{\mathrm{b}}$ Nanoscale Physics Research Laboratory, School of Physics and Astronomy, University of \\ Birmingham, Edgbaston, Birmingham, B15 2TT, UK \\ 'Irresistible Materials Ltd., Unit 4, Chelhydra Walk, Marina, Swansea, SA1 1YAG, UK \\ ${ }^{\mathrm{d} N a n o-C}$ Inc., 33 Southwest Park, Westwood, MA 02090, USA \\ ${ }^{\mathrm{e}}$ Laboratory for Micro and Nanotechnology, Paul Scherrer Institute, 5232 Villigen, Switzerland
}

\begin{abstract}
While the technological progress of Next Generation Lithography (NGL) steadily continues, further progress is required before successful insertion in high volume manufacturing is possible. A key issue is the development of new resists suitable to achieve higher lithographic resolution with acceptable sensitivity and line edge roughness. Molecular resists have been a primary focus of interest for NGL because they promise high resolution and small line edge roughness (LER), but no suitable resist candidate has emerged yet that fulfills all of the industry's criteria. We have previously shown first extreme ultraviolet lithography (EUVL) exposures for a new fullerene derivative based three-component negative tone chemically amplified resist with suitable properties close to or within the target range of the resist metrics as set out in the International Technology Roadmap for Semiconductors for 2016. Here we present the results of our efforts to optimize the EUVL performance of our resist system especially with regards to LER.
\end{abstract}

Keywords: EUV lithography, molecular resist, fullerene, chemically amplified resist

\section{INTRODUCTION}

With the advancement in next generation lithography (NGL) technology continuing, the progress in patterning capability is increasingly being limited by the lithographic performance of the photoresist. Current lithographic nodes already require linewidth roughness that is smaller than the radius of gyration of typical resist polymers [1]. Further progress in the development of new resists is needed to enable the commercial production of semiconductors at the sizes mapped for the future. Low molecular weight resists, such as fullerenes [2], triphenylenes [3], molecular glasses [4] and inorganic resists [5], have been a focus of interest for NGL because their small size promises high resolution and small line edge roughness (LER). But so far no resist candidate has emerged that fulfills all the industry's criteria. We have previously reported on first results of a new fullerene derivative based three-component negative tone chemically amplified resist for EUVL [6]. Here we present the results of a study on the influence of the process parameters on the EUVL performance of our fullerene derivative based resist system. Particular focus was placed on the correlation between process conditions and LER.

\section{EXPERIMENTAL}

Silicon substrates of $18 \mathrm{~mm}$ by $18 \mathrm{~mm}$ were prepared by dicing a $100 \mathrm{~mm}$-type, $<100>$-silicon wafer (Si-MAT) using a Disco DAD 321 wafer dicer. The substrates were cleaned using semiconductor grade chemicals (Puranal, Sigma Aldrich). After dicing, the substrates were immersed in isopropyl alcohol (IPA) and placed in an ultrasonic bath for 10 minutes. The samples were then rinsed in flowing deionised (DI) water for 1 minute (Purite Neptune, $18.2 \mathrm{M} \Omega \mathrm{cm}$ ) before being immersed in freshly prepared $\mathrm{H}_{2} \mathrm{SO}_{4}(95-98 \%): \mathrm{H}_{2} \mathrm{O}_{2}(30 \%)$ [1:1] for 10 minutes. After another 1-minute rinse in flowing DI water, the substrates were dipped for 3 minutes in a weak aqueous solution of hydrofluoric acid $(0.1$

*a.p.g.robinson@bham.ac.uk; phone+44(0)121414 4641

Advances in Patterning Materials and Processes XXXI, edited by Thomas I. Wallow, Christoph K. Hohle, Proc. of SPIE Vol. 9051, 905119 - (c) 2014 SPIE - CCC code: 0277-786X/14/\$18 · doi: 10.1117/12.2046268 
$-1 \%$ ) to form a hydrophobic surface, and finally a further 1-minute rinse in flowing DI water. They were then dried with nitrogen and used immediately for spin coating.

Resist formulation was done by mixing the fullerene derivative with a crosslinker and a photo acid generator at various ratios and concentrations in ethyl lactate. A fullerene containing underlayer has been developed that is pre-coated onto the chips to provide a suitable surface for resist spinning. After spin coating the samples received a post application bake. All exposures were performed using the XIL-II interference lithography tool at the Paul Scherrer Institute (PSI), Switzerland [7]. After exposure the samples received a post exposure bake and were then developed in suitable solvents.

Exposed samples were analyzed with a Zeiss Supra scanning electron microscope (SEM) in top-down view. Critical dimension (CD) and LER were calculated from the SEM images with the commercial software package SUMMIT. As the XIL tool does not allow direct measurement of the dose at the wafer, the exposure dose was measured with respect to an internal PSI reference resist calibrated against exposures of the same resist at the Intel MET [8].

\section{RESULTS}

In order to optimize the performance of our fullerene resist, we have undertaken an extensive study to find a set of process conditions that reduce the LER of the patterns. For optimization both the preparation of the resist and the resist processing was included. Care was taken to hold all other conditions constant for each parameter so that no two conditions were varied at the same time to avoid combinatorial overlap. But the system could not be fixed for all parameters, so that between different parameters the conditions might have changed (for example the fullerene derivative or the developer).

\subsection{Resist Formulation}

In this section the effect of the individual components of the resist material on the patterning performance was investigated. As it is a blended system, the constituents can be interchanged for different versions effortlessly without any additional chemical synthesis. The photo acid generator (PAG), triphenolsulfonium hexafluoroanimonate, was not changed, as from previous experiments it was seen to be the best choice for our system. The PAG loading level however was subjected to study.

\subsubsection{Fullerene Derivative}

The fullerene derivative version is still the most dominant factor for the patterning quality of the resist. As shown in figure 1 different variants show differences in photo speed and LER. The overall chemical structure of the derivative itself is the same in these variants, but modifications in the synthetic route yield widely varying patterning behavior. The chemistry of functionalizing the fullerenes is complex and challenging. Starting material, reaction byproducts and residues can be present after the reaction, so material purification is required. This is typically done with chromatography. Work to improve the synthesis is still ongoing and new versions are constantly created. Since the resist is an epoxy-based system, a comparison of the material without the fullerene derivative was conducted. It was seen that for the epoxy-only resist, the patterning quality was low and resolution limited to about $70 \mathrm{~nm}$ pitch with some modulation at $50 \mathrm{~nm}$ pitch. The addition of the fullerene derivative resulted in much improved patterning with examples shown in figure 2. For this study primarily features of $22 \mathrm{~nm}$ half pitch were analyzed as the data set at this resolution is much broader. However, the resist is capable of higher resolution (figure 3). An issue, which we observe here at smaller features, is a kind of pattern collapse. But it is not related to mechanical instability, as the aspect ratios are low. The lines start to snake and wiggle which we attribute to swelling. This presents the biggest challenge for our material to pattern with low LER at high resolution, as the analysis software translates this into higher numerical values while the lines themselves actually appear smooth. It is therefore a primary focus of the study to reduce the impact of this phenomenon. 


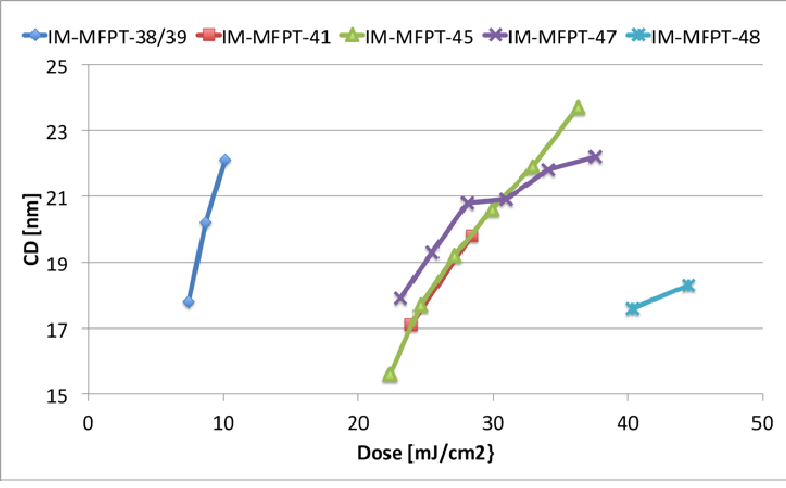

(a)

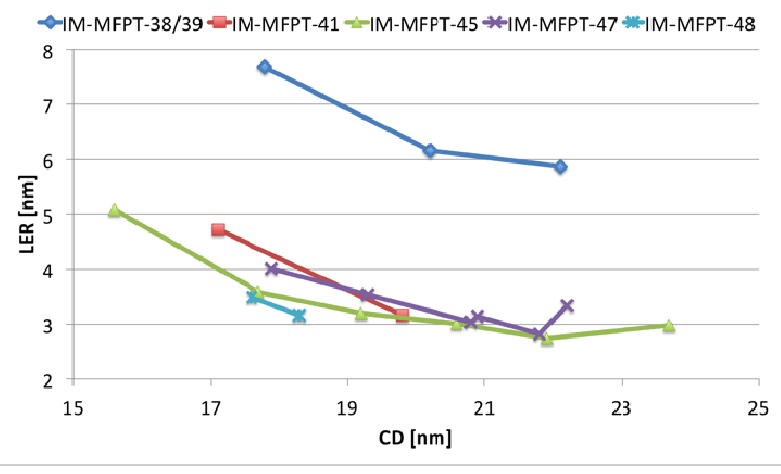

(b)

Figure 1 Comparison of fullerene derivatives for (a) sensitivity and (b) LER vs. critical dimension (CD)

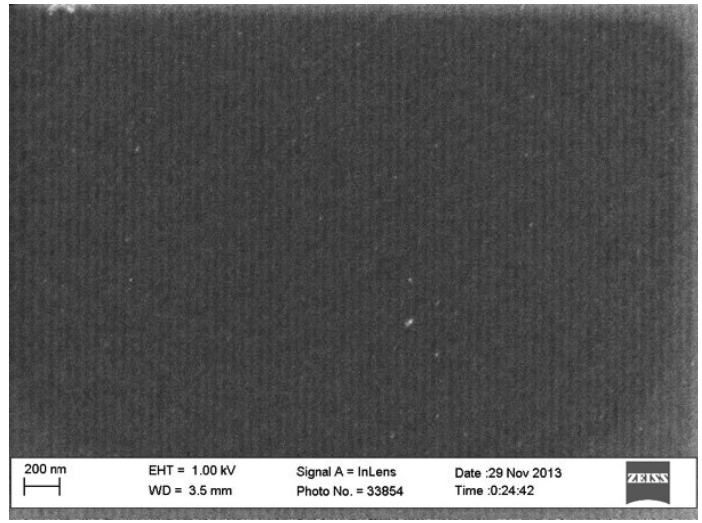

(a)

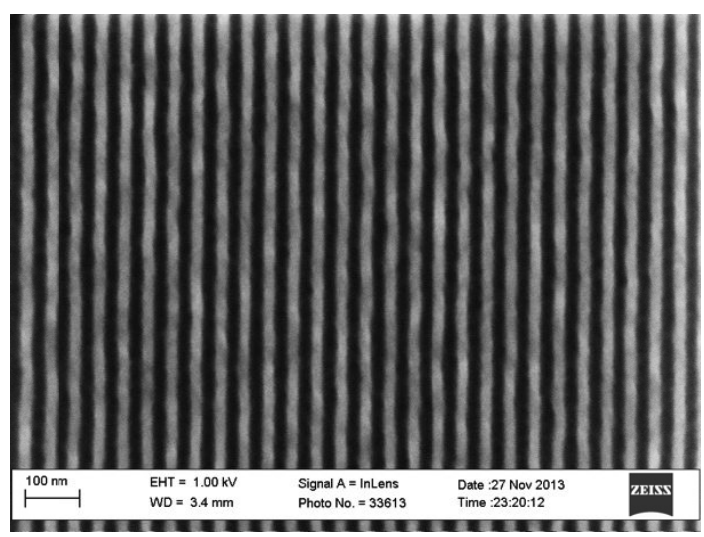

(c)

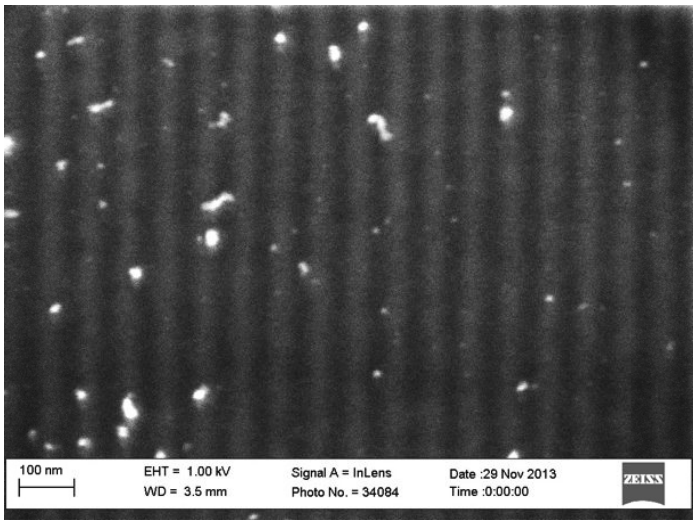

(b)

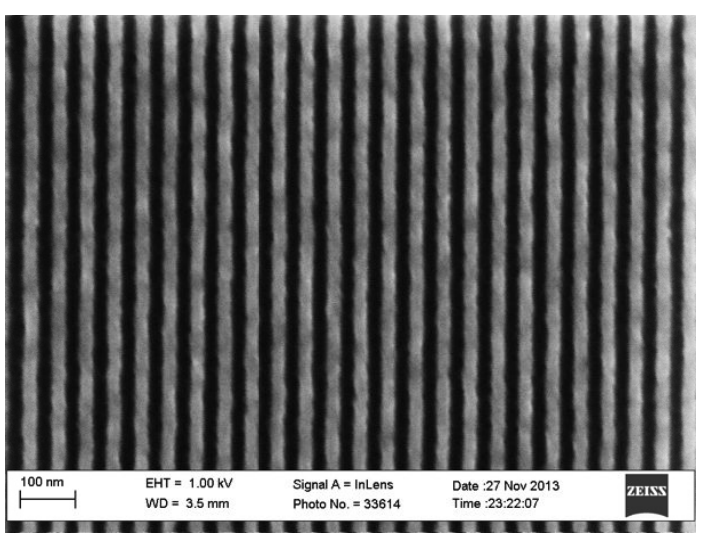

(d)

Figure 2 Resist performance without the fullerene [(a) $50 \mathrm{~nm}$ pitch \& (b) $70 \mathrm{~nm}$ pitch] and with fullerene derivative [(c) $44 \mathrm{~nm}$ pitch \& (d) $50 \mathrm{~nm}$ pitch] 


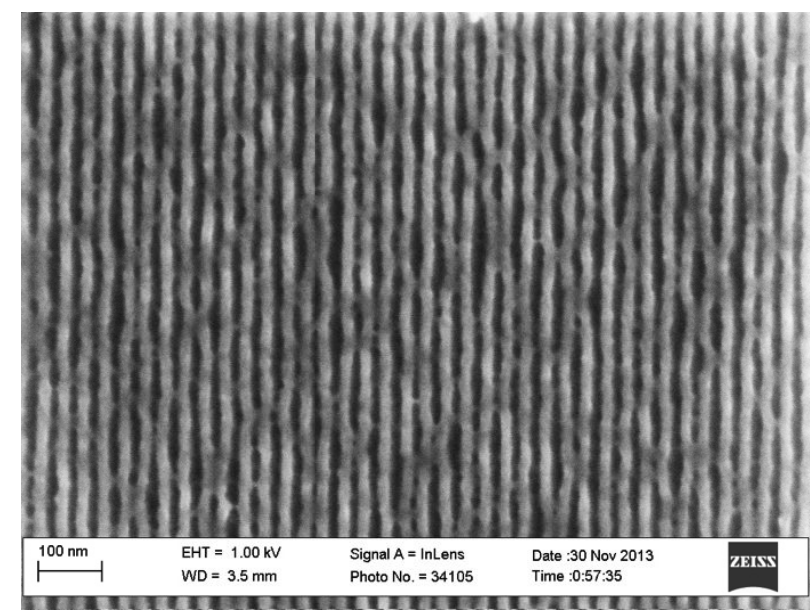

\subsubsection{Crosslinker}

Figure 3 SEM image of $16 \mathrm{~nm}$ features

One possibility for the swelling is that it might be caused by insufficient crosslinking. A variety of crosslinkers was tested with the resist. All are epoxies with either polymeric (CL01 - CL04) or molecular structure (CL05 - CL07). A mixture of crosslinkers was also tried with aim to provide a combination of different crosslinking lengths to improve the density and stability of crosslinks. As expected the choice of crosslinker had an influence on LER as well as sensitivity with CL02 and CL08 showing the best performance (figure 4). In addition they were the most successful in suppressing the snaking at higher resolution while some of the other materials did not pattern well at smaller feature size (figure 5).

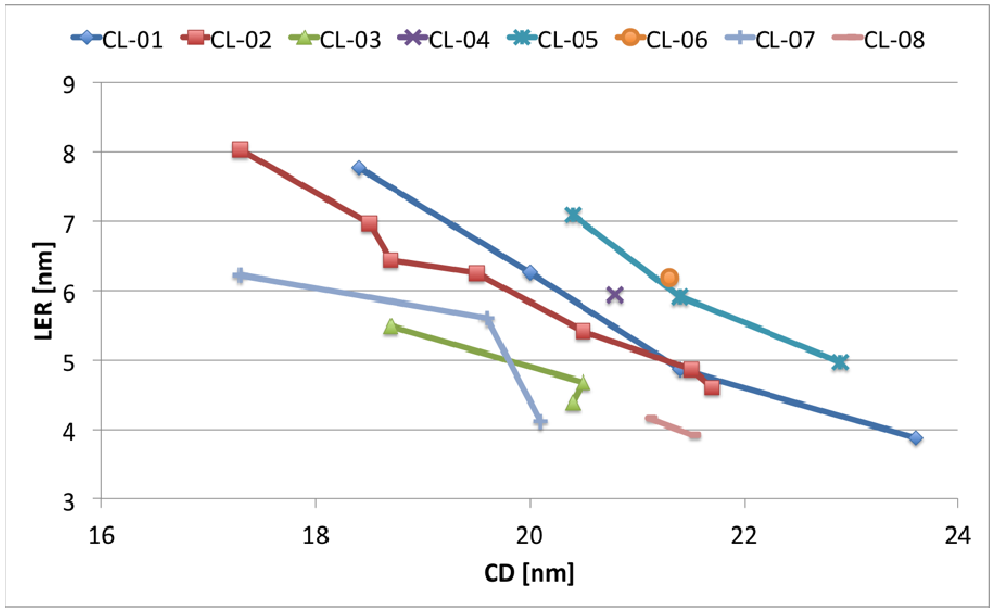

Figure 4 LER vs. CD for different crosslinkers

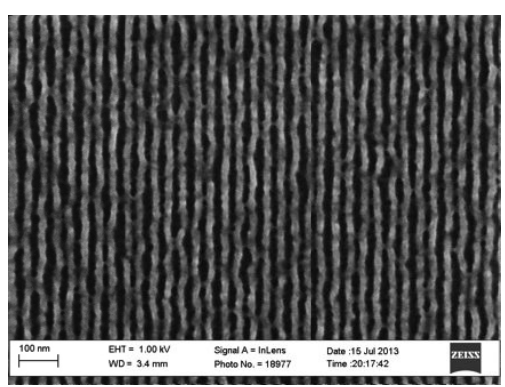

(a)

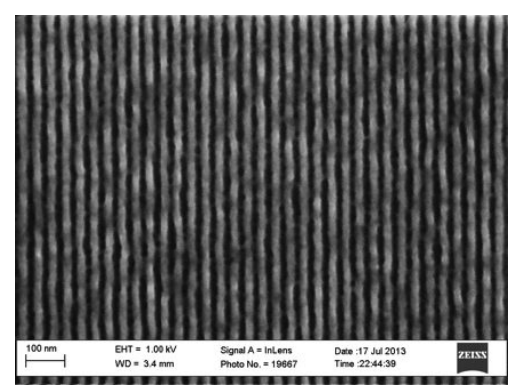

(b)

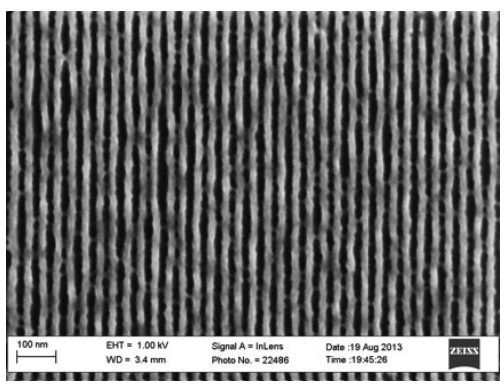

(c) 


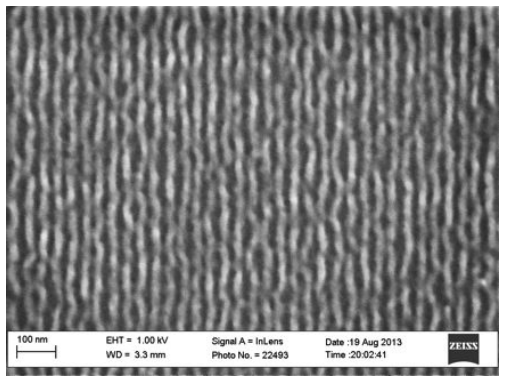

(d)

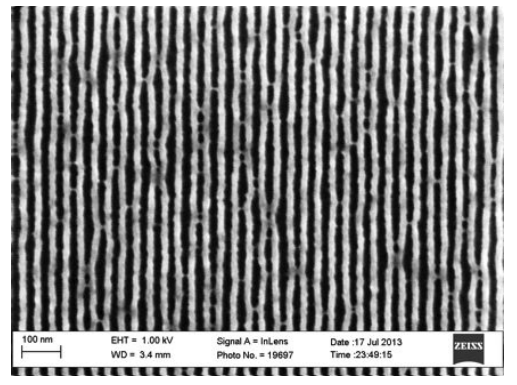

(e)

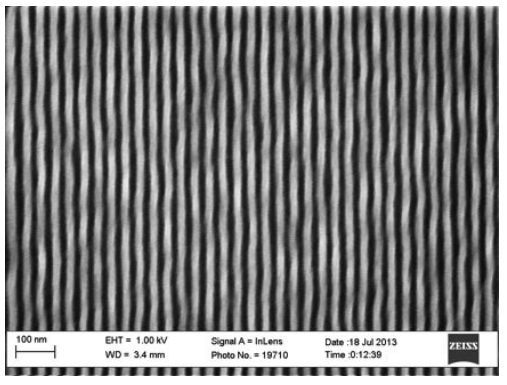

(f)

Figure 5 Patterning performance of different crosslinkers at $36 \mathrm{~nm}$ pitch: (a) CL-01, (b) CL-02, (c) CL-03, (d) CL-04, (e) CL-07 and (f) CL-08

\subsubsection{Mixing Ratio}

In the first instance the loading of PAG was decreased in anticipation of patterning improvement. If a high sensitivity material is chosen, a reduction of PAG should drive it toward a slower resist trading of sensitivity for LER. However we observe an immediate degradation in patterning quality (figure 6). In the next step a complete formulation analysis was undertaken. The fullerene amount was fixed and the proportions of crosslinker and PAG varied according to a statistical design matrix. We find that best results are achieved with a mixing ratio close to 1:2:1 (figure 7). The concentration of the resist solution was also investigated. With the spin speed fixed this leads to different film thicknesses and has an effect on the resulting patterns (figure 8). As mentioned earlier, the aspect ratio is not the critical issue here. The reduction in resist volume might be responsible for the suppression of snaking in the $15 \mathrm{~g} / \mathrm{L}$ solution, while $10 \mathrm{~g} / \mathrm{L}$ yielded a film too thin to pattern properly.

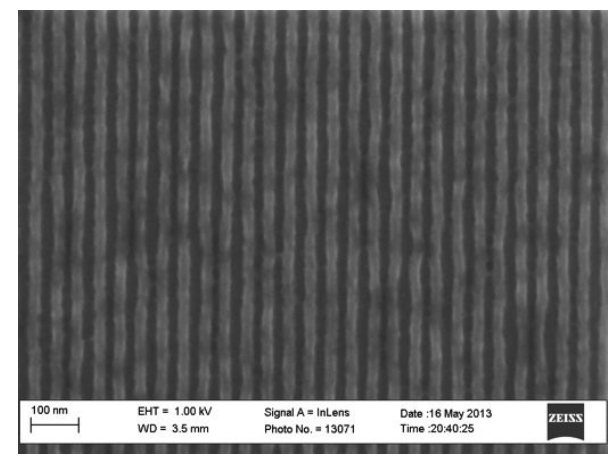

(a)

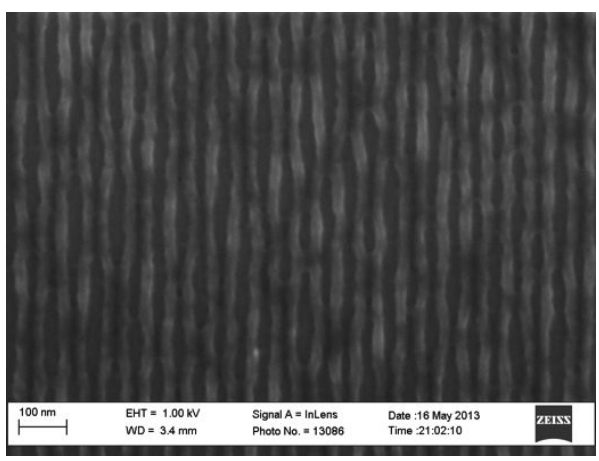

(c)

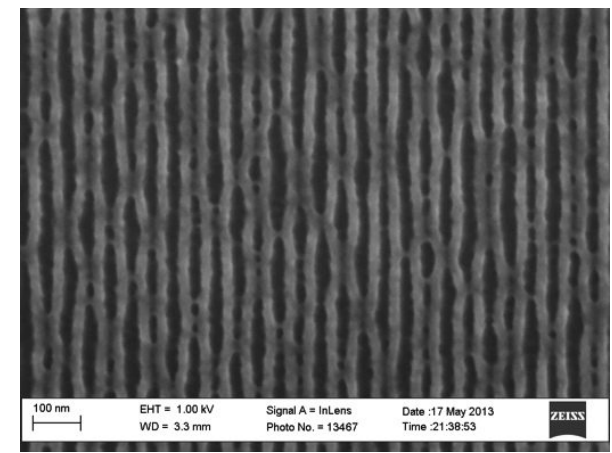

(b)

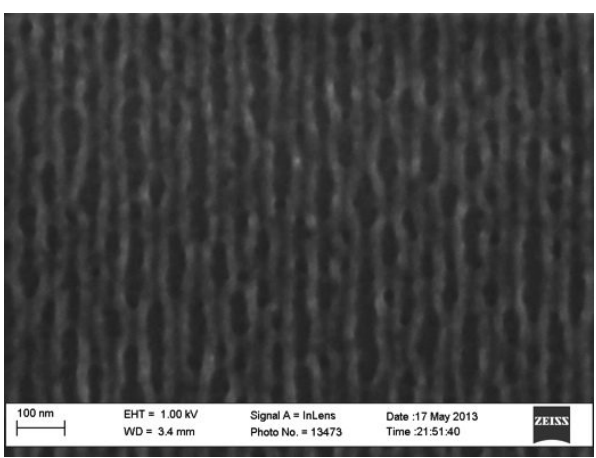

(d)

Figure 6 Variation of PAG loading: (a) 1:2:1, (b) 1:2:0.5, (c) 1:2:0.25 and (d) 1:2:0.1 


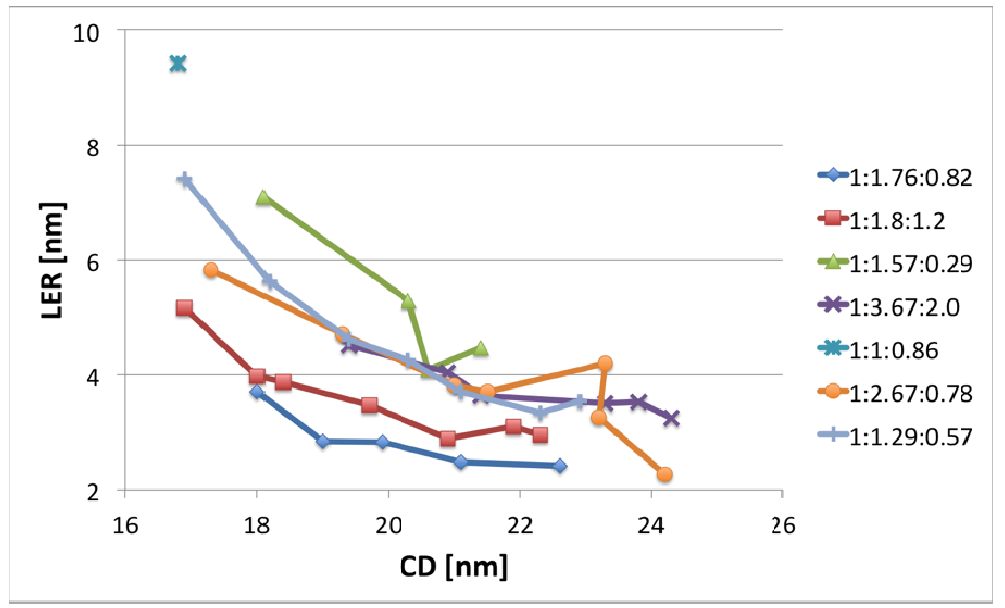

Figure 7 LER vs. CD for different resist compositions

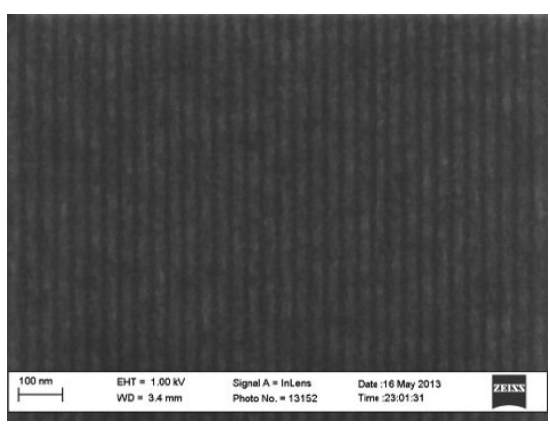

(a)

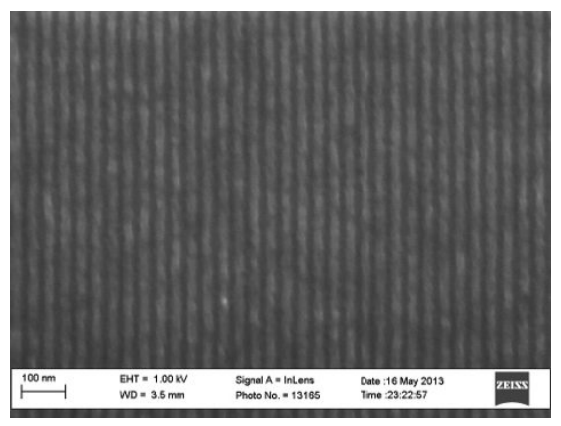

(b)

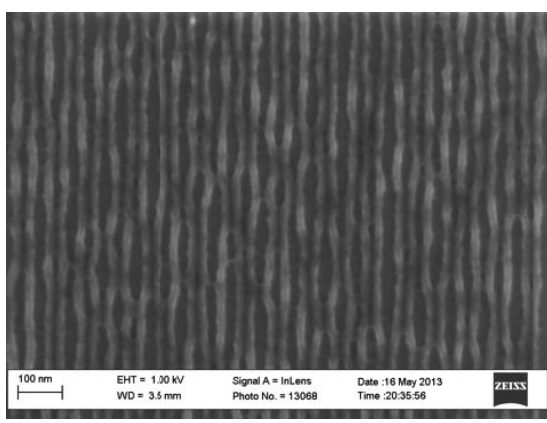

(c)

Figure 8 Patterning at different variations for $16 \mathrm{~nm}$ lines: (a) $10 \mathrm{~g} / \mathrm{L}$, (b) $15 \mathrm{~g} / \mathrm{L}$ and (c) $20 \mathrm{~g} / \mathrm{L}$

\subsubsection{Quencher}

It was been shown that triflates can effectively quench systems of cationic polymerization while bases are not suited for this purpose [9]. Addition of triphenolsulfonium triflate and nonaflate to the resist is shown to be a suitable route to reducing the LER of the material (figure 9).

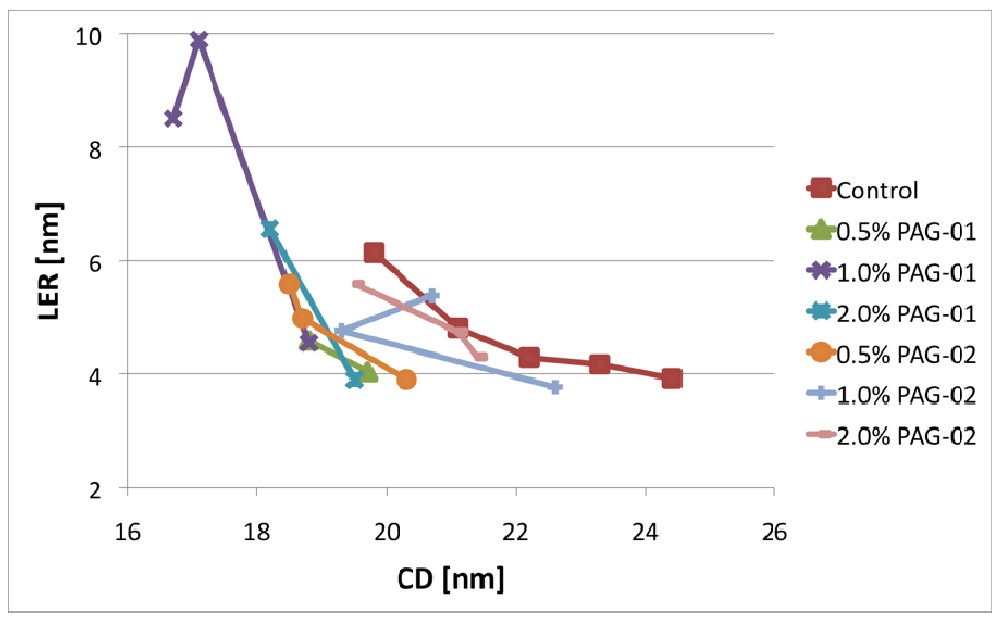

Figure 9 Effect of quencher on LER 


\subsection{Resist Processing}

In this section each of the process steps for exposing the material was investigated for its effect on the patterning behavior of the resist.

\subsubsection{Post application bake}

The PAB temperature was increased from $45^{\circ} \mathrm{C}$ in $15^{\circ} \mathrm{C}$ increments up to $120^{\circ} \mathrm{C}$. Bake durations tested were $1,2,3,5,8$ and 10 minutes. The bake duration had no measurable effect on the LER of the features, except that the highest chosen value resulting in degradation in the resist lines. Also the highest bake temperature showed signs of degradation. To our surprise we found that the bake temperature affected the sensitivity of the material with increasing temperature lowering the required dose-to-size (figure 10). In addition raising the temperature from $75^{\circ} \mathrm{C}$ to $105^{\circ} \mathrm{C}$ improved the LER as well.

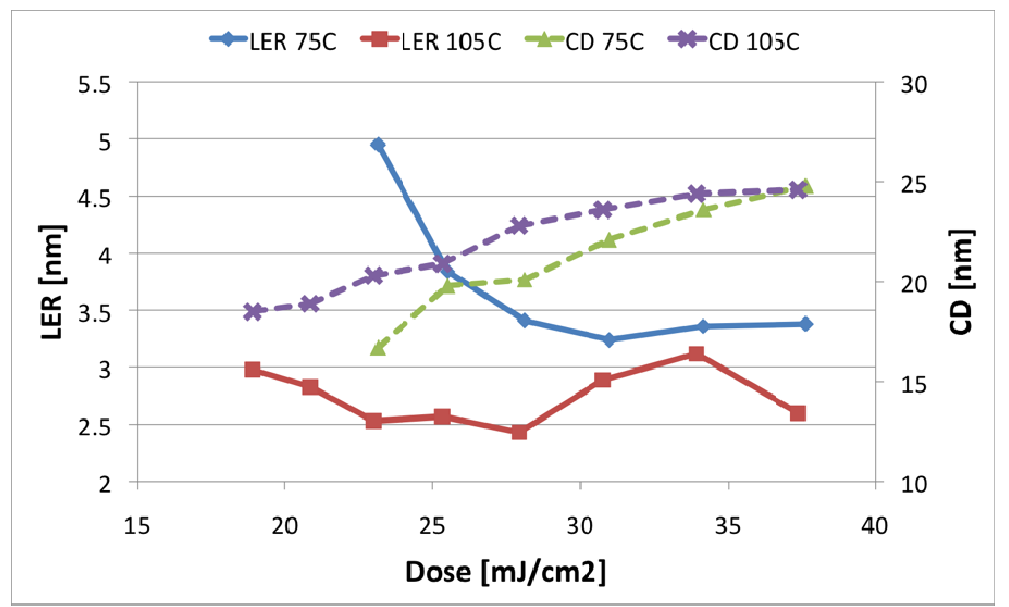

Figure 10 Comparison of sensitivity and LER for $\mathrm{PAB}$ at $75^{\circ} \mathrm{C}$ and $105^{\circ} \mathrm{C}$

\subsubsection{Post exposure bake}

The PEB temperature was also increased in $15^{\circ} \mathrm{C}$ increments from $45^{\circ} \mathrm{C}$ to $105^{\circ} \mathrm{C}$. The patterning result was independent of both bake temperature and duration. The reason is that even if no PEB is applied the exposure result is the same. The reaction must be catalyzed even at room temperature so that the material is already crosslinked before the sample is put on the hotplate.

\subsubsection{Development}

For development of the exposed resist our previous standard developer, a mixture of monochlorobenzene (MCB) and IPA, was compared to more industry accepted negative tone developers cyclohexanone, 2-heptanone and n-butyl acetate (nBA). All performed better then the MCB:IPA mix and caused less line wiggling with the nBA judged best from visible inspection of SEM images. Quantitative analysis was difficult due to variability in the data. The development time was also varied to see if shorter development could suppress the snaking. For very short times $(1 \mathrm{~s}, 5 \mathrm{~s})$ redeposition of resist material occurred, as manifested by particles on the lines. After 15 seconds the resist appeared clear and increasing the develop time did not result in further changes.

\subsubsection{Rinsing}

Three different rinse conditions were tested: no rinse, DI water and IPA. After applying no rinse or using DI water it was difficult to image the resist in the SEM leading to the conclusion that they are not viable options. They also did offer any visible improvement over the standard rinse solvent IPA. Furthermore the FIRM process was tested also tested as an additional step after the IPA rinse. The material has originally been developed for aqueous base development primarily to mitigate pattern collapse due to high aspect ratio, but we had the opportunity to also try it on our material. Surprisingly it was possible to improve LER of the fullerene resist with FIRM (figure 11) even though we use an organic developer. We presume the surfactant in the FIRM rinse to help with preventing redeposition of dissolved resist material. 


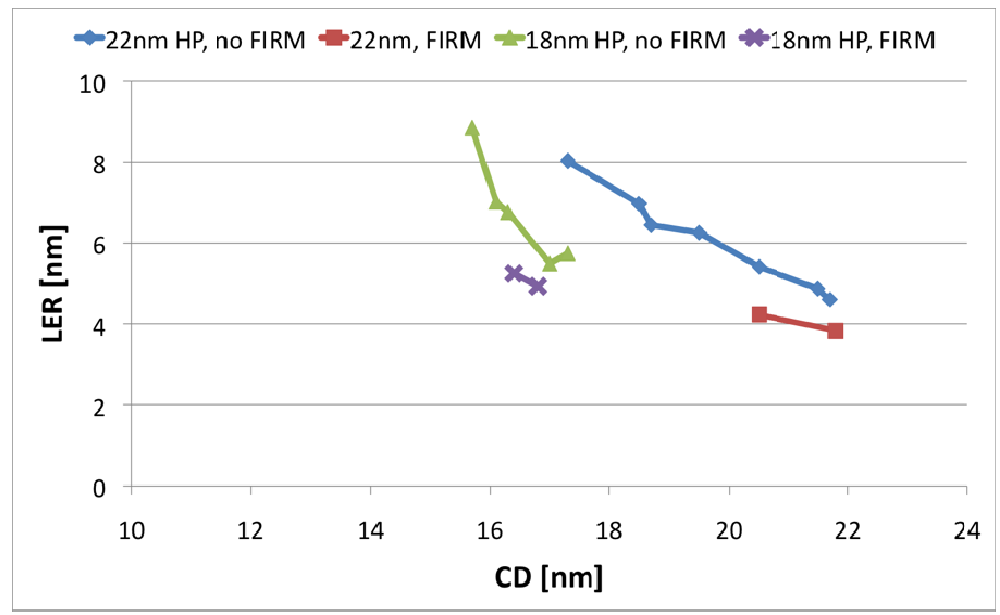

Figure 11 Effect of FIRM rinse on pattern quality

\subsubsection{Post development bake}

In an attempt to drive off excess solvent in the snaking lines and at least partially restore them a post development bake was applied to developed samples at various temperatures $\left(90-120^{\circ} \mathrm{C}\right)$. However it was found ineffective and actually introduce slight degradation in the patterns.

\subsubsection{Underlayer}

The last condition investigated was the use of underlayer. In addition to several commercially available underlayers, an in-house underlayer was also developed and all compared to patterning on bare silicon wafers. The in-house solution is a thin layer of the fullerene resist without the PAG and thermally crosslinked instead by baking on a hotplate at $300^{\circ} \mathrm{C}$. As shown in figure 12 the use of any underlayer reduced the sensitivity of the resist. Due to the lowered sensitivity on the underlayers, the samples patterned on the commercial underlayers were actually underexposed, so that their performance is difficult to analyze. From the data shown it might be possible that the underlayers work as well as the in-house solution - further investigation is necessary.

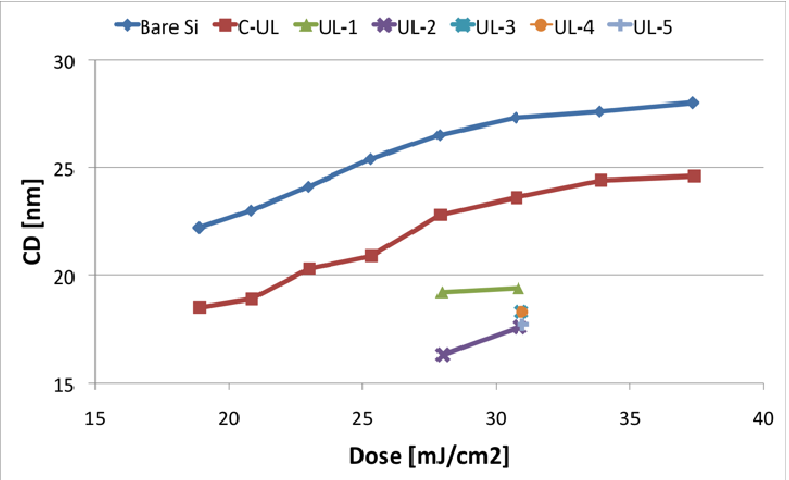

(a)

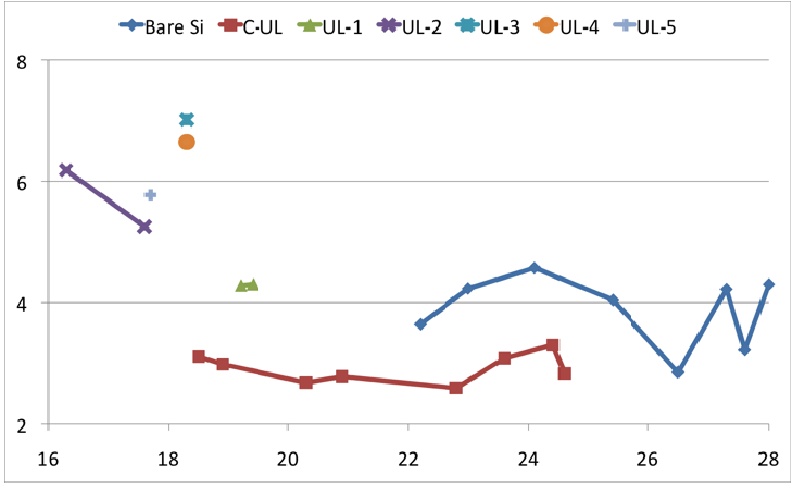

(b)

Figure 12 Effect of substrate on (a) sensitivity and (b) patterning performance

\section{CONCLUSION}

In summary we have presented results from our study of resist formulation and processing conditions on the EUVL performance of negative tone chemically amplified molecular fullerene resists. All relevant process parameters were studied and best conditions determined. Efforts on further improvement of fullerene derivate synthesis and crosslinking are continuing and we are optimistic that further increases in resolution and LER are possible. As the process conditions 
we report here were studied independently, in the next step we will look at combinations of the optimal conditions that we found, in order to investigate if they are cumulative in which case further gains may be expected. We are now at a stage where we can reliably and repeatedly pattern at $22 \mathrm{~nm}$ half pitch with an LER of around $2 \mathrm{~nm}$.

\section{ACKNOWLEDGEMENTS}

The authors thank the Engineering and Physical Sciences Research Council (EPSRC) for support of this project. The Disco DAD 321 wafer dicer used in this research was obtained, through Birmingham Science City: Creating and Characterising Next Generation Advanced Materials, with support from Advantage West Midlands (AWM) and part funded by the European Regional Development Fund (ERDF). The authors would like to thank Ms M. Vockenhuber from PSI for her assistance with the EUV exposures. Part of this work was performed at Swiss Light Source (SLS), Paul Scherrer Institute, 5232 Villigen, Switzerland.

\section{REFERENCES}

[1] Brainard R.L., Barclay G.G., Anderson E.H., Ocola L.E., "Resists for next generation lithography," Microelectron. Eng., Vol. 61-62, 707-715 (2002).

[2] Gibbons F.P., Robinson A.P.G., Palmer R.E., Diegoli S., Manickam M., Preece J.A., "Fullerene resist materials for the 32nm node and beyond," Adv. Funct. Mater., Vol. 18, 1977-1982 (2008).

[3] Zaid H.M., Robinson A.P.G., Palmer R.E., Manickam M., Preece J.A., "Chemical amplification of a triphenylene molecular electron beam resist," Adv. Funct. Mater., Vol. 17, 2522-2527 (2007).

[4] Lawson R.A., Tolbert L.M., Henderson C.L., "Single Component Molecular Resists Containing Bound Photoacid Generator Functionality," Proc. SPIE, Vol. 7273, 72733C (2009).

[5] Trikeriotis M., Krysak M., Chung Y.S., Ouyang C., Cardineau B., Brainard R., Ober C.K., Giannelis E.P., Cho K., "A new inorganic EUV resist with high-etch resistance," Proc. SPIE, Vol. 8322, 83220U (2012).

[6] Frommhold A., Yang D.X., McClelland A., Xue X., Ekinci Y., Palmer R.E., Robinson A.P.G., "Performance of negative tone chemically amplified fullerene resists in extreme ultraviolet lithography", J. Micro/Nanolith. MEMS MOEMS. 12(3), 033010 (2013).

[7] Päivänranta B., Langner A., Kirk E., David C., Ekinci Y., "Sub-10 nm patterning using EUV interference lithography," Nanotechnology Vol. 22, 375302 (2011).

[8] Ekinci Y., Vockenhuber M., Terhalle B., Hojeij M., Wang L., Younkin T.R., "Evaluation of resist performance with EUV interference lithography for sub-22 nm patterning," Proc. SPIE 8322, 83220W (2012).

[9] Henderson C.L., Lawson R.A., Tolbert L.M., "Molecular Resist Materials for EUVL Lithography: What Might Be Possible and How Do We Get There," 2009 International Workshop on EUV Lithography 